\title{
Quadrature amplitude modulation algorithm and its application of LTE system based on MIMO-OFDM platform Rongfang-Mei ${ }^{1}$, Baichuan-Cai ${ }^{2}$, Jianguo-Mei ${ }^{3}$
}

\author{
1.(Department of electronic information and control engineering, Yibin Vocational and Technical \\ College, Yibin Sichuan,644003)
2.( Department of electronic information and control engineering, Yibin Vocational and Technical College, Yibin Sichuan,644003)

\section{3. (Chongqing Municipal Health Technical School,banan chongqing ,401320)}

Keywords: broadband communication; ISI; distortion; LTE; MIMO

\begin{abstract}
In the design of LTE system, the multi input and multi output (MIMO) platform is taken as the basic configuration, high speed data transmission business is realized, due to multipath channel reorganization produced spatial focusing, intersymbol interference leads to channel imbalance, in order to improve the spectrum utilization rate, increase the system capacity, we need to increase the transmission power or bandwidth. The traditional method used orthogonal frequency division multiplexing algorithm for intersymbol interference suppression of LTE, since the carrier amplitude has negative signal distortion, it is unable to realize single week dynamic compensation. The effect is poor. A design method of LTE system is proposed based on MIMO-OFDM quadrature amplitude modulation QAM algorithm, the intersymbol interference suppression of broadband communication is taken, spectrum energy is calculated, and the input bit stream string is processed with conversion and modulation, harmonic weekly gain is produced. The communication signal is taken with OFDM decomposition, the turning negative signal sampling is obtained, the quadrature amplitude modulation of LTE system is obtained, and the algorithm is improved. Simulation results show that the algorithm is applied in the quadrature amplitude modulation and communication interference suppression of LTE system, it has god performance, it can optimize MIMO array distribution of LTE system, improve the impulse response gain of receiving array elements, and the quality of communication can be improved.
\end{abstract}

\section{Introduction}

With the rapid development of mobile communication network, $4 \mathrm{G}$ wireless cellular mobile communication has entered into people's lives, LTE and WiMAX, and 3GPP2 ultra mobile broadband (Ultra Mobile Broadband, UMB) are combined together, which called as $4 \mathrm{G}, 4 \mathrm{G}$ mobile communication network is established based on the LTE system, it can provide the voice and data communications with wireless networks, in the $4 \mathrm{G}$ Era, the mobile communication becomes full data network, LTE has maximum download rate with $100 \mathrm{Mbps}$ and upload $50 \mathrm{Mbps}$, it is better than $3 \mathrm{G}$ era. The core algorithm of WiFi, WiMAX and LTE downlink is DFT, it is built based on the MIMO-OFOM platform, and the FFT algorithm is used for data sampling and signal processing. Compared with WiMAX as the fixed wireless network technology, orthogonal frequency division multiplexing (OFDM) is used for signal transmission, Viterbi and Turbo accelerator are taken in advantage. But the WiMAX is from the IP technology, and LTE is derived from the mobile wireless communication technology of GSM/UMTS and LTE, 3GPP plans to use the OFDMA in the downlink, which is known as "the DFT extension OFDM")[1].

In broadband MIMO communication system, due to multi-path channel reorganization produced spatial focusing, intersymbol interference is produced, and channel imbalance is occurred, making the signal has severe non-linear distortion at the transmitting end, when the work safety scope of LTE scope cannot satisfy the quasi linear OFDM requirements, signal distortion is produced[2], and we need intersymbol interference suppression, distortion suppression and anti interference algorithm is researched, it has become the key to solve the problem of the related technology of MIMO. In the traditional method, the BPSK symbol distribution and digital modulation technology are used in mobile communication and antenna LTE system design, phase shift keying (PSK) and quadrature amplitude modulation (QAM) are the core technology in LTE system design, but the traditional method cannot effectively achieve the channel change tracking[3]. So it is unable to realize single week dynamic compensation. The communication performance is not good.

According to the problem as above, in this paper, a new design method of LTE system is 
proposed based on MIMO-OFDM quadrature amplitude modulation QAM algorithm, the intersymbol interference suppression of broadband communication is taken, spectrum energy is calculated, and the input bit stream string is processed with conversion and modulation, harmonic weekly gain is produced. The communication signal is taken with OFDM decomposition, the turning negative signal sampling is obtained, the quadrature amplitude modulation of LTE system is obtained, and the algorithm is improved. Simulation results show that the algorithm is applied in the quadrature amplitude modulation and communication interference suppression of LTE system, it has god performance, it can optimize MIMO array distribution of LTE system, improve the impulse response gain of receiving array elements, and the quality of communication can be improved, it will has good application value in practice.

\section{System model of MIMO broadband communication and problem description}

\section{Basic configuration of LTE system and system model}

Firstly, the system model of MIMO broadband communication is designed, multipath channel model of MIMO broadband communication system adopts distributed design of BPSK modulation symbols, in the process of system model and channel model design, the propagation loss is used to describe the signal communication transmission energy loss in the process, the ray model is used to describe the electromagnetic wave signal propagation loss[4], MIMO broadband communication channel are described as:

$$
T L=n \cdot 10 \lg r+\alpha r
$$

In the formula, TL is the propagation loss of MIMO-OFDM wireless communication channel (dB), $n$ is the propagation factor, $r$ is the attenuation process of MIMO-OFDM (m), $a$ is the absorption coefficient $(\mathrm{dB} / \mathrm{km})$; In the broadband communication process, due to the extended losses changes with the distance increases ${ }^{[5]}$, the absorption loss increases with the distance increases, so signal to noise ratio at the receiving end is:

$$
\mathrm{SNR}=S L-T L-(N L+D I)-10 \log \mathrm{W}
$$

In the formula, SL is launching MIMO-OFDM source level (dB), NL is interference range spectrum level of MIMO-OFDM $(\mathrm{dB} / \mathrm{Hz})$, DI is the directive gain $(\mathrm{dB}), \mathrm{W}$ is the system bandwidth $(\mathrm{Hz})$, according to the dispersion effect, the relationship of wave absorption $a$ and the MIMO broadband communications electromagnetic frequency $f(\mathrm{KHz})$ is:

$$
a=\frac{0.1 f^{2}}{1+f^{2}}+\frac{40 f^{2}}{4100+f^{2}}+2.75 \times 10^{-4} f^{2}+0.003
$$

In this paper, QAM modulation is used, and $C_{\mathrm{n}}=a_{n}+j b_{n}$ is the QAM modulation symbol of $\mathrm{n}$ subcarriers. DC gain of the horizon MIMO-OFDM channel distributed at the receiving end is:

$$
s(v)=\int_{0}^{v} \sin \left(\frac{\pi}{2} x^{2}\right) d x
$$

Thus, the impulse response of the multipath channel can be obtained as:

$$
|s(f)|=A \sqrt{\frac{1}{2 k}}\left\{\left[c\left(v_{1}\right)+c\left(v_{2}\right)\right]^{2}+\left[s\left(v_{1}\right)+s\left(v_{2}\right)\right]^{2}\right\}
$$

In the formula, $A(t)$ is the signal amplitude, $f_{0}$ is the initial frequency, $k=B / T$ is the frequency modulation slope, and B is the FM signal bandwidth. Through the analysis of MIMO broadband communication channel, multipath effect is easy to cause the MIMO broadband communication, in this paper, the orthogonal frequency division multiplexing (OFDM) is improved as MIMO-OFDM for MIMO broadband communication, so that the signal interference still can reliably receive, amplitude negative signal distortion can be suppressed, dynamic compensation of distortion is realized, the system model structure of MIMO broadband communication system is shown in Figure 1. 


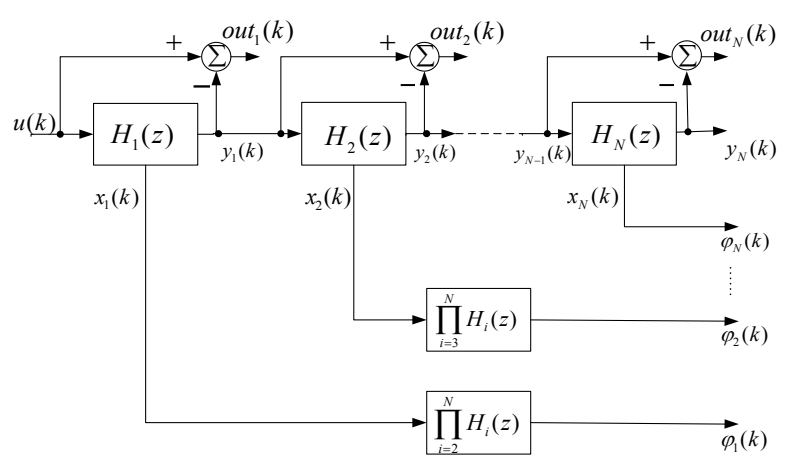

Figure 1. Diagram of MIMO broadband communications system with linear feedback block

In the figure, including two tap delay filter: a filter (FFF) is a forward, another is the inverse filter (FBF), the fractional spaced equalizer is equivalent to the best linear matched filter, best linear receiver is designed, wherein, a matched filter is formed with equalizer microwave symbol rate sampling, while the FSE is not less than the Nyquist rate sampling, the best combination can be achieved with the matching filter and spaced equalizer characteristics, through the above analysis, the construction of broadband MIMO communication system model is obtained, it can provide a model foundation for the realization of broadband communication intersymbol interference suppression algorithm improvement.

\section{Generation of multipath intersymbol interference and OFDM signal decomposition}

According to the above analysis, the MIMO-OFDM signal will generate the time extension after broadband communication channel propagation, in MIMO broadband communication, it will produce spatial focusing, then MIMO-OFDM filter is coupled into the transmission channel, in order to make the MIMO-OFDM data frame satisfy the block fading, it needs to input bit stream string and conversion and modulation, multipath intersymbol interference is generated, some channel multipath components or the existence of phase offset are calculated, phase offset is added in the multipath components, then, the channel impulse response can be expressed as:

$$
h\left(\tau_{i}, t\right)=\sum_{i=1}^{N_{m}} a_{i}(t) e^{j \theta_{i}(t)} \delta\left(t-\tau_{i}(t)\right)
$$

In digital communication system, insert a tunable filter to correct and compensate the system characteristics, to reduce the effects of intersymbol interference. The filter compensation is called as equalizer. Digital communication system with an equalizer can be equivalent to the model shown in Figure 2.

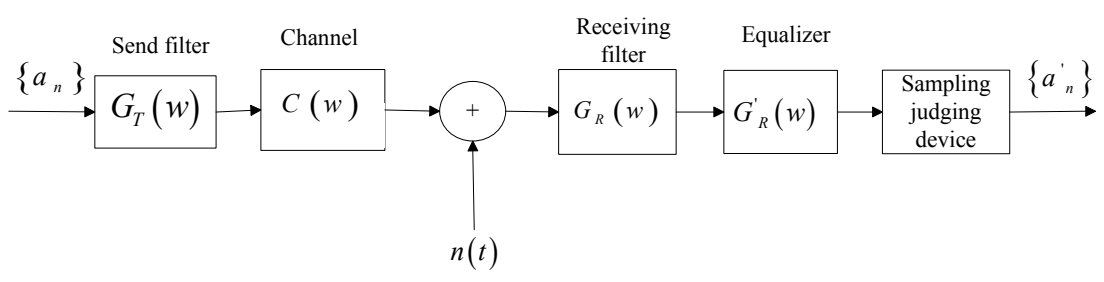

Figure 2 Communication system with an equalizer

The equalizer usually adopts $T / 2$ tap interval, frequency response of FSE can be expressed as:

$$
C_{T^{\prime}}(f)=\sum_{k=-K}^{K} c_{k} e^{-j 2 \pi f k T^{\prime}}
$$

Where, $c_{k}$ is the tap coefficient of the equalizer, $T^{\prime}=M T / N$. Thus, $C_{T^{\prime}}(f)$ can beyond the Nyquist frequency to the frequency of received signal spectrum, and the frequency $f=N / M T$, the received signal can be taken with equalization processing. Spectrum after equalization can use formula as follow to calculate:

$$
C_{T^{\prime}}(f) Y_{T^{\prime}}(f)=C_{T^{\prime}}(f) \sum_{n} x\left(f-\frac{n}{T^{\prime}}\right) e^{j 2 \pi\left(f-\frac{n}{T^{\prime}}\right) \tau_{0}}=C_{T^{\prime}}(f) X(f) e^{j 2 \pi f \tau_{0}}|f| \leq \frac{1}{2 T^{\prime}}(8)
$$

Where, $X(f)$ is the input analog signal spectrum with limited bandwidth, $Y_{T^{\prime}}(f)$ is the sampling signal frequency spectrum. Because of the LTE nonlinear distortion, data vector of QAM modulation on the current compensation and voltage recovery in the opposite direction, 
In order to obtain broadband communication channel of real signal, to suppress the intersymbol interference, $C_{\mathrm{n}}$ need to be further expanded, the OFDM signal meet the HSP sequence is obtained obtained after IFFT, it is:

$$
\mathrm{x}_{k}=\sum_{n=0}^{N-1} C_{n} \cdot e^{j 2 \pi k n / N} k=0,1, \cdots, N-1
$$

Through the positive and negative signal decomposition, signal sampling of time domain signal is obtained, through demodulation and parallel / serial conversion, the output bit stream is obtained, next step, the LTE nonlinear distortion of one cycle control is used to provide unipolar signal model, and the QAM algorithm is improved.

\section{Quadrature amplitude modulation (QAM) control of LTE system and intersymbol interference suppression algorithm}

\section{Quadrature amplitude modulation of LTE system}

In broadband MIMO communication OFDM system, ${ }^{w(k)}$ isused to describe the MIMO-OFDM photodiode output signal, using the characteristics of OFDM signal, PTS and Clipping algorithm are combined, the decision feedback equalizer is designed as follows, shown in Figure 3.

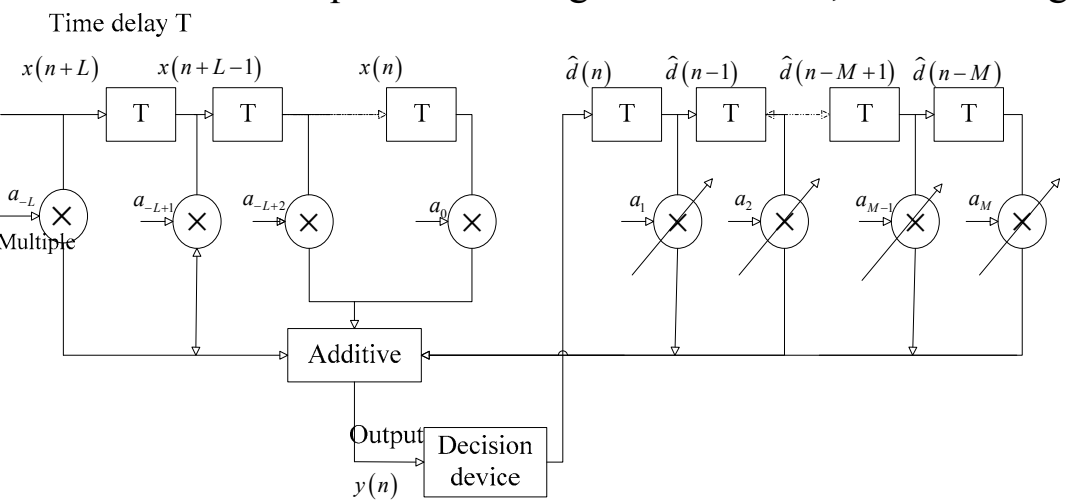

Figure 3. Decision feedback equalizer of LTE system

When the system subcarrier modulation of the lower order is smaller, and the number of subcarriers is small, proper weight coefficient $b_{v}$ is selected, which satisfies:

$$
\left\{b_{1}, b_{2}, \cdots, b_{v}\right\}=\underset{\left\{b_{1}, b_{2}, \cdots, b_{v}\right\}}{\arg \min \min }\left(\max \left|\sum_{v=1}^{V} b_{v} \bullet x_{v}\right|^{2}\right)
$$

In high speed broadband MIMO communication system, $\arg \min (\bullet)$ is expressed as minimum value when the decision condition is satisfied, MIMO-OFDM wireless channel serial data stream is cited with clipping noise, $\tilde{x}$ is the optimal choice, weighted coefficient is:

$$
\left.\left\{b_{1}^{\prime}, b_{2}^{\prime}, \cdots, b_{v}^{\prime}\right\}=\underset{\left\{b_{1}, b_{2}, \cdots, b_{v}\right\}}{\arg m} \sum_{v=1} b_{v=1} b_{x_{v}<0,1 \leq n \leq N}\left|\sum_{v=1}^{V} b_{v} \bullet x_{v}\right|^{2}\right)
$$

Get $x^{\prime}=\sum_{v=1}^{v} b_{v}^{\prime} x_{v}, x^{\prime}$ is taken with polar decomposition, a distributed LTE platform MIMO-OFDM source spectrum peak estimation is obtained. Assumed $C_{0}=C_{N / 2}=0, C_{N-n}=C_{n}^{*}$, and $n=0,1,2 \cdots N / 2-1$, therefore, the intersymbol interference suppression of broadband communication is taken, spectrum energy is calculated, and the input bit stream string is processed with conversion and modulation.

\section{Improved algorithm}

The traditional method used orthogonal frequency division multiplexing algorithm for intersymbol interference suppression of LTE, since the carrier amplitude has negative signal distortion, it is unable to realize single week dynamic compensation. The effect is poor. A design method of LTE system is proposed based on MIMO-OFDM quadrature amplitude modulation QAM algorithm. The key technology to implement the algorithm described as below: In broadband communication systems, based on MIMO-OFDM platform, the multipath interference signal and the receiver signal are obtained: 


$$
y(t)=r \bullet h(t) \otimes s(t)+z(t)
$$

Where, $s(t)$ is the sending signals, $h(t)$ is the channel impulse response, power conversion efficiency $r=1, \int_{-\infty}^{+\infty}|h(t)|^{2} d t=1$, because the channel noise, multipath intersymbol interference exists, resulting in a received signal may be negative, one cycle control method is used for suppressing the noise, and the QAM algorithm is improved, the negative signal wave signal is expressed as:

$$
\tilde{y}_{k}^{+}=\left\{\begin{array}{ll}
y_{k}^{+}, & y_{k}^{+} \geq 0 \\
0, & y_{k}^{+}<0
\end{array}, \quad \tilde{y}_{k}^{-}= \begin{cases}y_{k}^{-}, & y_{k}^{-} \geq 0 \\
0, & y_{k}^{-}<0\end{cases}\right.
$$

According to the Bussgang theorem, the cumulative distribution function (CDF) of the received signal power is:

$$
F_{\text {power }}(z)=\int_{0}^{z} \frac{1}{\sqrt{2 \pi}} p^{-1 / 2} e^{-p / 2}
$$

The communication signal is taken with OFDM decomposition, the turning negative signal sampling is obtained, the MIMO-OFDM power is emitted into the MIMO-OFDM wireless communication channel by means of weighted coefficient value, the appropriate set is selected, it can enhance the probability to obtain small amplitude negative signals, the performance of the QAM algorithm improved, the channel gain is improved, visibly, the method is effective.

\section{Simulation experiments and results analysis}

In order to test the performance of the proposed algorithm, simulation experiment is taken, MIMO broadband communication uses OSRAMSFH 4230 high power, LTE platform is taken as a broadband communication components, the signal to reverberation ratio is $-30 \mathrm{~dB}$, communication environment is very poor.

System parameters are set, sampling is conducted in the symbol $1 / 3$ and $2 / 3$ respectively, the order of feedforward filter is 24, tap spacing of feedforward filter is $1 / 2$ symbol interval, the iteration step are 0.01 , length is 4000011 , feedback coefficient $c_{20} \sim c_{0}$ is 100000000000000001001 . 5 channel impulse response of MIMO broadband communication are simulated, and the simulation results are shown in Figure 4.

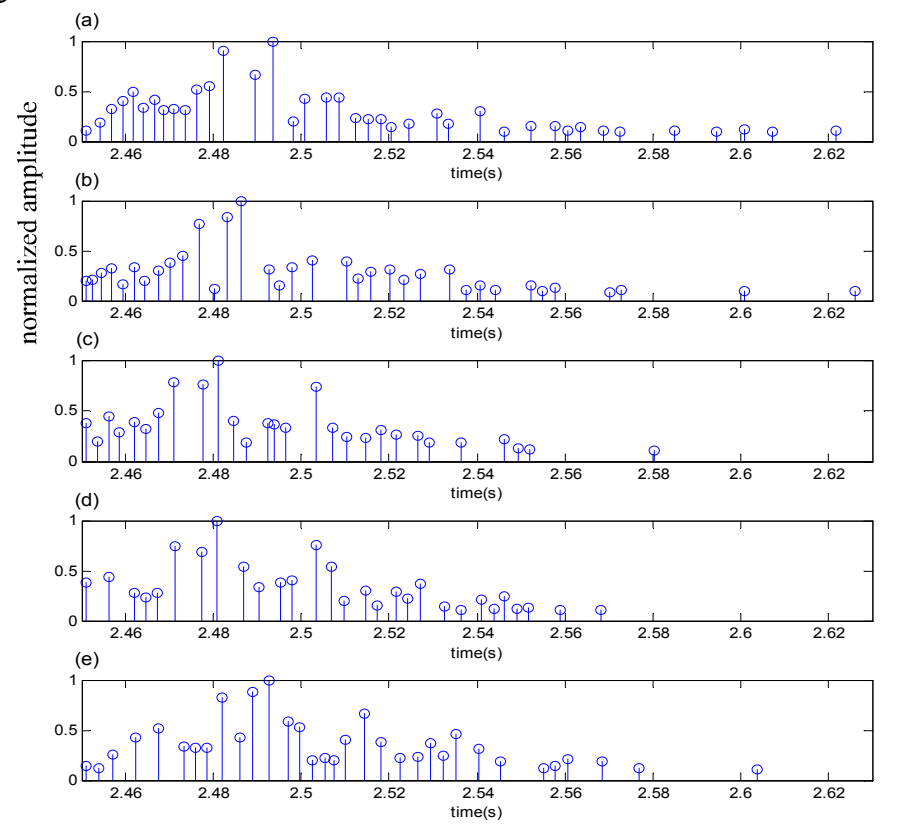

Figure 4. 5 channel impulse response of MIMO broadband communication

BER is taken as the evaluation standard, and with different array element number, the BER with the change of the number of array elements is shown in Figure 5. 


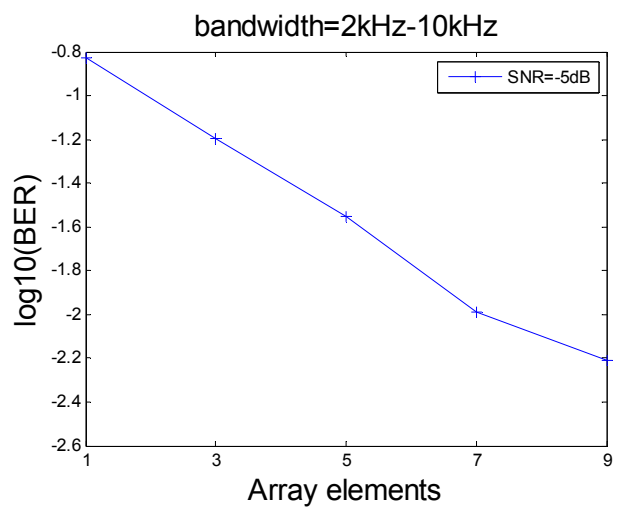

Figure 5. BER with the change of the number of array elements

From the simulation result, it shows that, when SNR $=0 \mathrm{~dB}$, BER can be reduced to below 10-3, it shows that the new QAM algorithm can suppress intersymbol interference effectively, it has superior performance of MIMO broadband communication, the communication quality is improved.

\section{Conclusions}

In this paper, a MIMO-OFDM quadrature amplitude modulation QAM algorithm is proposed based on LTE system, the intersymbol interference suppression of broadband communication is taken, spectrum energy is calculated, and the input bit stream string is processed with conversion and modulation, the quadrature amplitude modulation of LTE system is obtained, and the algorithm is improved. Simulation results show that the QAM algorithm can suppress intersymbol interference effectively, it has superior performance of MIMO broadband communication, the communication quality is improved. It will have good application value in practice.

\section{References}

[1] Rahman M S, Haque M M, Kim K D. Indoor Positioning by LTE Visible Light Communication and Image Sensors[J]. Inter-national Journal of Electrical and Computer Engineering ( IJECE) , 2011,1(2): 161-170.

[2] Lee Y U, Kavehrad M. Long-range indoor hybrid localization system design with visible light communications and wireless net-work[C]// Photonics Society Summer Topical Meeting Series, 2012: 82-83.

[3] Yang Bo, Wang Zhijie. Research on Performance of Communication Equilibrium Based on PTRM and DS Technology[J]. Bulletin of Science and Technology, 2014,30(2):212-214.

[4] GUO Xiao-yan. Simulation and Analysis on Uncertain Attenuation Property of Underwater Acoustic Signal for Oil Field Pipe[J]. Computer Simulation, 2014,31(3):118-121.

[5] ZHANG Ren-shang. Network Intrusion Detection System Based on Expert System and Neural Network[J]. Computer Simulation. 2012; 29(9): 162-165. 